\title{
Epileptic Seizure, Cataract, and Tongue Atrophy during the 8 Years after Electrical Brain Injury
}

\author{
Takeo Kuwabara ${ }^{1}$, Takao Fukushima ${ }^{1}$, Kunihiko Makino ${ }^{1}$ and Hiroshi Kondo ${ }^{2}$
}

\begin{abstract}
As a delayed neurologic complication following a high-voltage electrical injury, motor neuron disease-like spinal cord injury has often been reported. However, epileptic seizure as a delayed complication of electrical brain injury has not been reported. We report a 32-year-old man, who developed epilepsy 8 years after electrical brain injury. His electroencephalogram (EEG) recordings showed focus on the right side, in which the electrical current passed during the accident eight years earlier. He experienced cataract during these 8 years. On examination, the right side of his tongue was found to be atrophic, and he was unaware when it had started. Systemic EMG examination revealed neuropathic changes in both (interossei dorsalis) IODs, the right biceps, right tongue, and right masseter muscles.
\end{abstract}

Key words: electrical brain injury, delayed neurologic complication, symptomatic epilepsy, motor neuron disease, traumatic cataract

(Inter Med 48: 1179-1182, 2009)

(DOI: 10.2169/internalmedicine.48.2008)

\section{Introduction}

Symptoms of electrical injury are diverse, from instantaneous cardiac arrest due to a high-voltage current to transient unpleasantness caused by a low-voltage current. Loss of consciousness, confusion, convulsive seizure, and poor recall immediately after a high-voltage injury are well known as immediate influences on the central nervous system (CNS) (1). Cataractogenesis following electrical trauma with a latency period varying from immediately after injury to a few years have been reported in sporadic cases $(2,3)$. As delayed neurologic complications after a high-voltage electrical injury, motor neuron disease-like spinal cord injury has often been reported (4-6). Convulsive seizure at the time of electrical shock, and epilepsy as a sequel of closed head injury caused by a fall is known well, however, to our knowledge, epileptic seizure as a delayed complication of electrical brain injury without closed head injury has not been reported.

In this report, we describe a case which presented with epileptic seizure, cataract, and tongue atrophy in the 8 years following a high-voltage electrical brain injury.

\section{Case Report}

A 23-year-old man electrician accidentally touched a high-voltage electrical wire $(5,800$ volts, A.C. $50 \mathrm{~Hz})$, while working on a utility pole on September 2, 1999. He lost consciousness and experienced a brief episode of tonic convulsion. He did not fall from the height. He was taken to a local hospital immediately. While being transported by ambulance, he regained consciousness. At the hospital, he was found to be fully alert and in no acute distress. However, he could not remember the accident. An electrical injury was diagnosed with an entry wound on the right occipital area of the skull and an exit wound at the medial part of both thighs. He had a third-degree burn over the right occipital area and third-degree burns on the thighs. He had a good regular pulse of 94/min. His ECG and chest X-ray showed no abnormalities. Computed tomography (CT) of the head was normal.

Laboratory investigations showed a normal blood count as well as normal electrolyte, blood sugar, liver enzyme, creatine kinase (CK), and myoglobulin levels. He underwent treatment for his burns. In early convalescence during his

${ }^{1}$ Department of Neurology, Niigata Prefectural Shibata Hospital, Shibata City and ${ }^{2}$ Brain Disease Center Agano Hospital, Agano City Received for publication January 5, 2009; Accepted for publication March 17, 2009

Correspondence to Dr. Takeo Kuwabara, kuwabara@sbthp.jp 


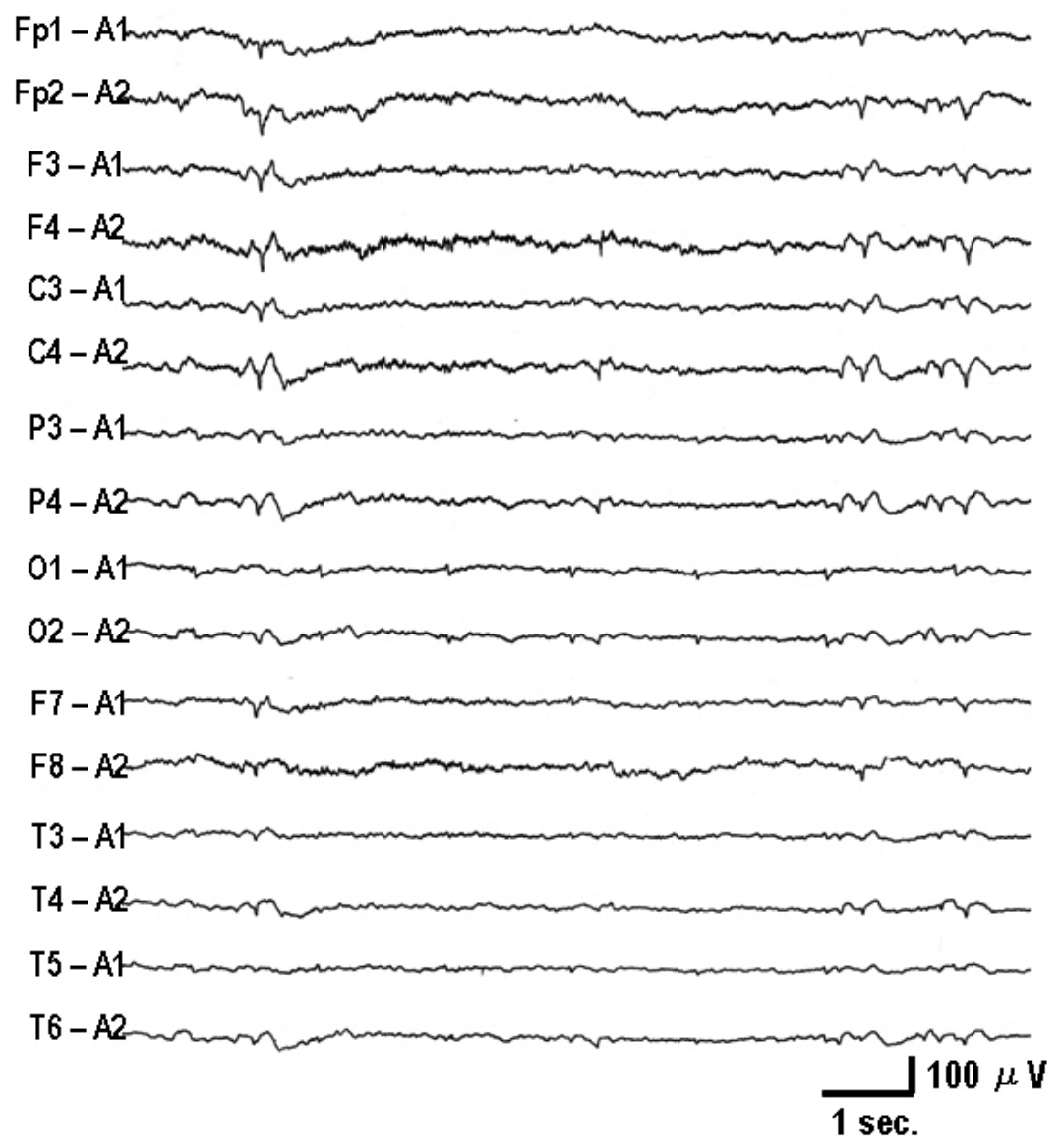

Figure 1. An electroencephalogram (EEG) at the second seizure. EEG recording showed right mid-saggital slowing with small theta elements and positive sharp waves.

hospital stay, he suffered from headaches, insomnia, and general malaise for a couple of weeks. He was discharged from hospital without sequelae and was able to resume work 3 months after the accident.

Three years after the accident, when he was 26 years old, he noticed a progressive decrease in vision of the right eye over the preceding year. Slit lamp examination and Scheimpflug imaging confirmed anterior and posterior subcapsular lenticular opacities in the right eye. Examination of the left eye showed no abnormality. He was diagnosed with juvenile cataract in his right eye. Phacoaspiration and posterior chamber intraocular lens implantation were performed under topical anesthesia in the right eye.

Afterwards, he resumed daily life without inconvenience. However, when he was 31 years old, he experienced a generalized tonic-clonic convulsion during sleep, and was taken to an emergency clinic. Magnetic resonance imaging (MRI) showed no abnormality. Laboratory investigation showed a normal blood count and electrolyte, blood sugar, and liver enzyme levels. An electroencephalogram (EEG) at the second seizure showed right mid-saggital slowing with small theta elements and positive sharp waves (Fig. 1). He was diagnosed with focal epilepsy. He was successfully treated by the oral administration of $200 \mathrm{mg}$ of phenytoin and additional $80 \mathrm{mg}$ of phenobarbital.

One year after the first convulsive seizure, he was referred to our hospital to confirm the cause of the epileptic seizure on June 26, 2008, when he was 32 years old.

On general physical examination, healed burn scars were recognized over the right occipital area of the skull and the medial part of both thighs. Neurological examination revealed that the patient was cooperative and well oriented. Although the shape of the right pupil was irregular due to the eye surgery in 2002, the pupils of both eyes responded identically to a light stimulus. The movement of both eyes was full and smooth. Cranial nerves other than the right XIIth nerve were intact. Liguals was slightly impaired, but he was unaware when it had started. He did not complain of difficulty in swallowing. The right half of the tongue was atrophied, but there were no fasciculations (Fig. 2). His grip strength was $62.3 \mathrm{~kg}$ for the left hand and $65.5 \mathrm{~kg}$ for the right, and muscle strength of the arms and legs was normal. Deep tendon reflexes were absent. Deep and superficial sensation was normal. The rest of the neurological examination was unremarkable.

A nerve conduction study of the 4 limbs, including Fwaves and H-reflexes, showed nothing of note. Sensory evoked potential (SEP) was normal. Electromyography (EMG) was performed involving the left and right biceps, 1 st interossei dorsalis (IOD), quadriceps femoris, tibialis anterior, masseter, and tongue muscle. EMG of the left and right 1 st IOD and right biceps showed neuropathic changes: 


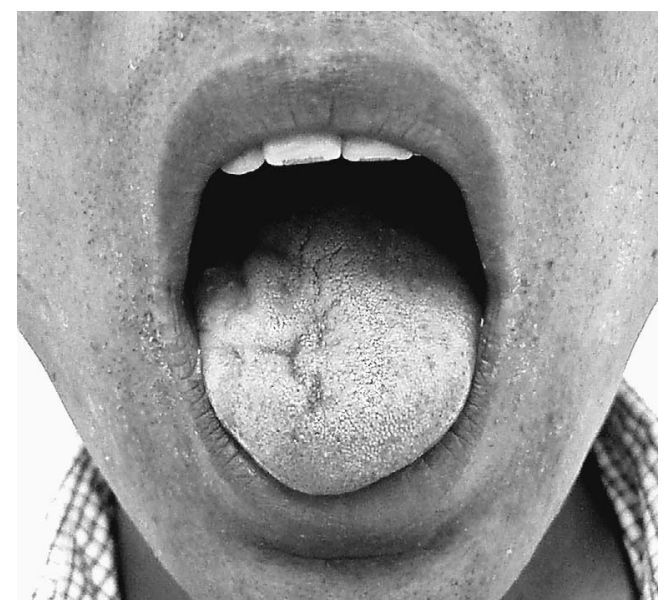

Figure 2. Hemiatrophy of the tongue. The right half of the tongue was atrophied, but there were no fasciculations.

a reduced interference pattern, long duration, high amplitude $\&$ polyphasia. Changes of the right biceps were milder than those of both 1st 1ODs. Samplings from both legs were normal. Study of the cranial nerve area showed neuropathic changes over the right masseter and tongue muscles. There were no abnormal findings on the left side. No EMG samplings demonstrated findings suggesting active neuronal cell damage, such as fibrillation potentials or positive sharp waves. MRI of the cervical and lumbar spine revealed no abnormalities.

\section{Discussion}

The present patient showed unilateral cataract, hemiatrophy of the tongue, and epileptic seizure in the 8 years after electrical injury. EMG samplings from the arms, tongue, and face showed neuropathic changes. Changes were localized only to the left and right 1 st 1 ODs, right biceps, right tongue, and right masseter muscles. We failed to demonstrate the systemic neuropathic changes usually shown in motor neuron disease. Also, the samples generated findings that do not correspond to the existence of an active process.

As neural tissue is a better conductor of electricity than other tissues such as skin, skull, and bones, neurologic injury after an electrical burn is not uncommon (1, 7-9). Central nervous system damage can manifest either immediately or after a latent period of months or years $(1,8)$. Common immediate neurologic manifestations are characterized by a loss of consciousness, seizures, headaches, agitation, mania, confusion, amnesia, impaired memory, emotional lability, learning impairment, changes in character, acute motor and sensory neuropathies, and reflex sympathetic dystrophy (1, $7,8)$. Cranial nerve symptoms such as optic atrophy, transient papilledema, pupillary abnormality, bilateral fifth nerve hypoesthesia, dysphasia, and facial paralysis have also been reported as immediate neurological presentations $(10,11)$.

Delayed neurologic disorders include basal ganglia disorders $(1,8)$, and spinal cord injury with and without recovery $(4,5,10,12)$. Spinal cord injury is by far the most common of the permanent as well as transient complications, and it resembles either progressive muscular atrophy or motor neuron disease $(10,12)$. Some cases showed a latency of more than 10 years (12).

Epileptic seizure is uncommon as a delayed neurologic complication (1). In the present case, EEG revealed epileptogenic foci in the right hemisphere, which was thought to have constituted the main routes of current passage through the brain.

As the conductibility of blood vessels is better than that of neural tissue, the blood vessels constitute the main routes for the passage of currents through the brain. So, the genesis of neurologic injuries is suggested to be primarily vascular (11). Over the course of in vivo electrical conduction, electrical energy transforms into heat, thus compensating for the resistance of tissue. The resultant heat loss causes injury in the vascular intima and adventitia, which leads to the delayed degeneration of very small-sized blood vessels and resultant ischemic injury to brain tissue after some latent period $(5,10,11)$. Cryptogenic lesions such as scar, a tangle of blood vessels, or any other abnormality in the brain that could interfere with its delicate electrical workings may have caused epileptic seizure after 8 years (13). The secondary intimal proliferation of blood vessels and the resultant ischemia is thought to have caused the delayed degeneration of motor neurons through a mechanism similar to delayed radiation damage $(10,11)$.

Cases of electrical injury in Japan are decreasing after peaking in the 1960s. However, about 60 accidents still occur each year (14). Here, we presented the appearance of symptomatic epilepsy, in which the focus was thought to be on the side in which the electrical current passed during the accident eight years earlier. In the area thought to have constituted the main routes for the passage of currents, we demonstrated localized asymptomatic damage at the motor neuron level; we noted neuropathic hemiatrophy of the tongue, and pointed out neuropathic changes in both hands. Whether these changes occurred during the accident, or some time after a latent period still remains uncertain.

In conclusion, even in patients who seem to show a favorable outcome after electrical brain injury, we should consider the possibility of symptomatic epilepsy in the future. Also, we need to conduct further neurological examinations and testing to elucidate occult neurological complications in those who otherwise seem to exhibit no sequelae.

\section{References}

1. Silversides J. Neurological sequelae of electrical injury. Can Med Assoc J 91: 195-196, 1964.
2. Grewal DS, Jain R, Brar GS, Grewal SP. Unilateral cataract: Scheimpflug imaging and review of the literature. J Cataract Re- 
fract Surg 33: 1116-1119, 2007.

3. Chaudhuri Z, Pandey PK, Bhatia A. Electrical cataract: A case study. Ophthalmic Surg Lesers 33: 166-168, 2003.

4. Breugem CC, Van Hertum W, Groenevelt F. High voltage electrical injury leading to a delayed onset tetraplegia, with recovery. Ann NY Acad Sci 888: 131-136, 1999.

5. Jafari H, Couratier P, Camu W. Motor neuron disease after electric injury. J Neurol Neurosurg Psychiatry 71: 265-267, 2001.

6. Tashiro K, Inoue I, Ohyagi Y, et al. A case of motor neuron syndrome with onset 9 months after electrical injury. Rinsho Shinkeigaku (Clin Neurol) 40: 732-735, 2000 (in Japanese).

7. Grube BJ, Heimbach DM, Engrav LH, Copass MK. Neurologic consequences of electrical burns. J Trauma 30: 254-258, 1990.

8. Cherington M. Central nervous system complications of lightning and electrical injuries. Semina Neurol 15: 233-240, 1995.

9. Liao WC, Lin JT. High-voltage electrical brain injury. J Trauma 64: 843-845, 2008

10. Farrell DF, Starr A. Delayed neurological sequelae of electrical injuries. Neurology 18: 601-606, 1968.

11. Ko SH, Chun W, Kim HC. Delayed spinal cord injury following electrical burns: a 7-year experience. Burns 30: 691-695, 2004.

12. Gallagher JP, Talbert OR. Motor neuron syndrome after electric shock. Acta Neurol Scand 83: 79-82, 1991.

13. Lamy C, Domingo V, Semah F, et al. Early and late seizures after cryptogenic ischemic stroke in young adults. Neurology 60: 400404, 2003.

14. Annual Report from Japanese Ministry of Health, Labor and Welfare. 2006 (in Japanese).

(C) 2009 The Japanese Society of Internal Medicine http://www.naika.or.jp/imindex.html 